\title{
Renal Denervation for the Treatment of Ventricular Arrhythmias: A Systematic Review and Meta-Analysis
}

\author{
Gabriela Prado ${ }^{1}$, Felix Mahfoud ${ }^{2}$, Renato Lopes $^{3}$, Dalmo Moreira ${ }^{1}$, Rodolfo Staico ${ }^{1}$, Lucas \\ Damiani $^{4}$, Christian Ukena ${ }^{5}$, and Luciana Armaganijan ${ }^{1}$ \\ ${ }^{1}$ Instituto Dante Pazzanese de Cardiologia \\ ${ }^{2}$ Universitätsklinikum des Saarlandes und Medizinische Fakultät der Universität des \\ Saarlandes \\ ${ }^{3}$ Duke University \\ ${ }^{4}$ Hospital do Coracao \\ ${ }^{5}$ Saarland University Hospital and Saarland University Faculty of Medicine
}

November 4, 2020

\begin{abstract}
Introduction Ventricular arrhythmias (VAs) are a major cause of morbidity and mortality in patients with heart disease. Recent studies evaluated the effect of renal denervation (RDN) on the occurrence of VAs. We conducted a systematic review and metaanalysis to determine the efficacy and safety of this procedure. Methods and results A systematic search of the literature was performed to identify studies that evaluated the use of RDN for the management of VAs. Primary outcomes were reduction in the number of VAs and implantable cardioverter-defibrillator (ICD) therapies. Secondary outcomes were changes in blood pressure and renal function. Ten studies (152 patients) were included in the meta-analysis. RDN was associated with a reduction in the number of VAs, ATP (antitachycardia pacing), ICD shocks and overall ICD therapies of 3.53events/patient/month $(95 \% \mathrm{CI}=-5.48$ to -1.57$), 2.86$ events/patient/month $(95 \% \mathrm{CI}=-4.09$ to -1.63$), 2.04$ events $/$ patient/month $(95 \% \mathrm{CI}=-2.12$ to -1.97$)$, and 2.68 events/patient/month $(95 \% \mathrm{CI}=-3.58$ to -1.78$)$, respectively. Periprocedural adverse events occurred in $1.23 \%$ of patients and no significant changes were seen in blood pressure or renal function. Conclusions In patients with refractory VAs, RDN was associated with a reduction in the number of VAs and ICD therapies, and was shown to be a safe procedure. KEYWORDS: Renal denervation, ventricular arrhythmias, implantable cardioverter-defibrillator, antitachycardia pacing, ICD shocks.
\end{abstract}

\section{Hosted file}

MANUSCRIPT.pdf available at https://authorea.com/users/372970/articles/490771-renaldenervation-for-the-treatment-of-ventricular-arrhythmias-a-systematic-review-and-metaanalysis 